REVISTA INTERNACIONAL DE CIENCIAS DEL DEPORTE International Journal of Sport Science

doi:10.5232/ricyde2008.01001

Rev. int. cienc. deporte

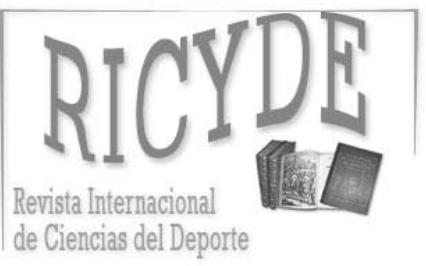

International Journal of Sport Science VOLUMEN IV. AÑO IV

Páginas:1-12 ISSN:1885-3137

No 10 - Enero - 2008

\title{
Relación entre la fuerza máxima en squat y acciones de salto, sprint y golpeo de balón. \\ Relationship among maximal strength in squat exercise, jump, sprint and kicking ball performance.
}

\author{
Daniel Juárez Santos-García \\ Fernando Navarro Valdivielso \\ Rosa María Aceña Rubio \\ José María González Ravé \\ Alfredo Arija Blázquez \\ Víctor Muñoz Fernández-Arroyo
}

Laboratorio de Entrenamiento Deportivo. Facultad de Ciencias del Deporte de Toledo/UCLM

\section{Resumen}

El objetivo del presente estudio fue analizar las posibles relaciones entre la fuerza máxima en squat y acciones explosivas de salto, sprint y golpeo de balón. Para ello, se contó con una muestra formada por estudiantes de Ciencias del Deporte $(\mathrm{N}=16 ; 19,6 \pm 1,7$ años $)$ de sexo masculino (con escasa experiencia en el entrenamiento de fuerza), que llevaron a cabo un test de 1 RM en squat (calculándose el peso levantado, la fuerza media total y la fuerza dinámica máxima), tests de salto vertical (SJ, CMJ y CMJa), un test de sprint de $20 \mathrm{~m}$ (con mediciones de tiempo cada $5 \mathrm{~m}$ ), y un test de golpeo con el pie donde se calculaba la velocidad del balón. Se encontraron coeficientes de correlación moderados (entre 0,52 y 0,67 ) y significativos de las medidas de fuerza máxima y las alturas de salto, mientras que los coeficientes obtenidos entre los tiempos de sprint y dichas medidas de fuerza máxima se situaron entre $-0,06$ y $-0,32$, no siendo ninguno de ellos significativo; al igual que tampoco fueron significativos los coeficientes de correlación de las medidas de fuerza máxima y la velocidad de tiro, se situaron entre 0,25 y 0,34 . Por tanto, la relación entre la fuerza máxima en squat y la altura de salto en sujetos con escasa experiencia en el entrenamiento de fuerza resultó moderada, mientras que no parece existir relación con el tiempo de sprint y la velocidad de tiro.

\section{Abstract}

The purpose of the present study was to analyze the possible relationships between maximal strength in squat exercise and jump, sprint and kicking ball actions. Sixteen males sport science students $(19,6 \pm 1,7$ years), with little experience in strength training, performed a 1RM in squat exercise (from which lifted weight, the total average- and maximal-dynamic strength was assessed), vertical jump (SJ, CMJ and $\mathrm{CMJa}$ ), a $20 \mathrm{~m}$ sprint test (with time measurement every $5 \mathrm{~m}$ ), and a kicking ball test where the velocity of the ball was calculated. Moderate $(0,52-0,67)$ and significant correlations were observed between maximal strength measures and jump ability. However, the correlations obtained between maximal strength and the sprint times ranged between $-0,06$ and $-0,32$, not being statistically significant. Correlations of 0,25 to 0,34 ( $n$. s.) were observed between maximal strength and ball velocity. In conclusion, the relationship between maximal strength in squat exercise and jump ability in subject with little experience in strength training was moderate, whereas no relationships were observed between maximal strength and the sprint time or ball speed. Therefore, other aspects, such as kicking and/or running technique, should be considered as possible performance predictors in this population.

Palabras clave: fuerza máxima, squat, salto, sprint, golpeo de balón.

Key words:maximal strength, squat, jump, sprint, ball kick.

Correspondencia/correspondence: Daniel Juárez Santos-García

Laboratorio de Entrenamiento Deportivo. Facultad de Ciencias del Deporte de Toledo. Universidad de Castilla la Mancha

E-mail: djuarezsg@yahoo.es 


\section{Introducción}

L a fuerza, mediante sus diversas manifestaciones, juega un papel esencial en una gran cantidad de disciplinas deportivas. Desde un punto de vista deportivo, se puede definir la fuerza como la manifestación externa (fuerza aplicada) que se hace de la tensión interna generada en el músculo o grupo de músculos en un tiempo determinado (González Badillo, 2000; González Badillo \& Ribas, 2002: 14); o como la capacidad de un músculo o grupo de músculos determinados para generar una fuerza muscular bajo unas condiciones específicas (Siff \& Verkhoshansky, 2000: 20).

La programación tradicional del entrenamiento de fuerza teniendo como objetivo fundamental la mejora de la fuerza explosiva, que se puede definir como la relación entre la fuerza producida (manifestada o aplicada) y el tiempo necesario para ello (González Badillo, 2000; González Badillo \& Ribas, 2002), se ha basado, de forma general, en el entrenamiento en primer lugar de la fuerza dinámica máxima, que sería la expresión máxima de fuerza cuando la resistencia sólo se puede desplazar una vez, o se desplaza ligeramente o transcurre a muy baja velocidad en una fase del movimiento (González Badillo, 2000; González Badillo \& Ribas, 2002), para, posteriormente, realizar una fase de conversión de esta fuerza hacia la fuerza explosiva y potencia (Bompa, 1999, , 2003; Bosco, 2000; Graham, 2002; Stone, O'Bryant, \& Garhammer, 1981; Wathen, Baechle, \& Earle, 2000).

Hay autores que han afirmado que la fuerza máxima es la cualidad que más influye en el rendimiento en potencia (Schmidtbleicher, 1992), considerando la existencia de una asociación entre la fuerza máxima y el aumento de la velocidad máxima del mismo movimiento (Bührle \& Schmidtbleicher, 1977; Häkkinen, Komi, \& Tesch, 1981; Hoff \& Almasbakk, 1995; Kaneko, Fuchimoto, Toji, \& Sney, 1983; Manno, 1999; Wenzel \& Perfetto, 1992). Sin embargo, Bosco (2000), opina que tener grandes valores de fuerza máxima o de fuerza dinámica máxima no es un requisito imprescindible para la obtención de buenos resultados en muchos deportes. A pesar de ello, tener un nivel óptimo de fuerza máxima y de fuerza dinámica máxima es fundamental para poder desarrollar elevados gradientes de fuerza explosiva. Otros autores indican que si no es necesario el desarrollo de una gran fuerza máxima, y prima el desarrollo de una gran velocidad, la fuerza máxima carece de importancia (Siff \& Verkhoshansky, 2000; Voigt \& Klausen, 1990; Young \& Bilby, 1993).

En este sentido, los coeficientes de correlación hallados entre el 1RM en sentadilla y la altura de salto vertical han sido diversos. Estos valores están influidos por las características de la muestra y su nivel de entrenamiento, ya que parece ser que los sujetos menos entrenados pueden mostrar coeficientes de correlación más elevados (Baker, 1996, 2001).

En un trabajo de investigación (Alegre, 2004) se observó una correlación de 0,57 $(\rho<0,05)$ entre los cambios en la altura en SJ y los cambios en $1 \mathrm{RM}$ en $1 / 2$ squat tras un programa de entrenamiento de 13 semanas, realizando 3 sesiones por semana, en estudiantes de Ciencias del Deporte, no habiendo encontrado otros autores relación 
entre estos cambios ( $r=0,11$; n.s.) en atletas expertos que entrenaron 3 veces por semana durante 12 semanas (Baker, Wilson, \& Carlyon, 1994). Uno de los ejercicios que se realizaron durante el programa de entrenamiento en estos dos estudios fue el de squat; sin embargo, en ninguno de ellos se llevaron a cabo ejercicios de salto, si bien en la investigación de Alegre (2004) se empleó el ejercicio de extensión de tobillos con una barra de pesas sobre los hombros. Sin embargo, en otro estudio con sujetos entrenados, en concreto futbolistas de élite (Wisloff, Castagna, Helgerud, Jones, \& Hoff, 2004), se encontró un coeficiente de correlación alto $(r=0,78 ; \rho<0,05)$ entre la altura de salto vertical y el peso levantado en 1 RM en $1 / 2$ squat.

En cualquier caso, siguiendo la opinión recogida por otros autores (Moss, Refsnes, Abildgaard, Nicolaysen, \& Jensen, 1997), parece que la fuerza máxima es también una variable importante para el rendimiento con cargas ligeras. De todas formas, la relación entre el peso levantado en squat y la capacidad de salto no parece estar del todo clara, ya que hay estudios donde se han encontrado correlaciones bajas y no significativas entre estas dos acciones (Cronin \& Hansen, 2005).

En relación a la velocidad de carrera, en otro trabajo de investigación (Wilson, Lyttle, Ostrowski, \& Murphy, 1995) se observó un coeficiente de correlación moderado ( $\mathrm{r}=$ 0,$62 ; \rho<0,05$ ), en 15 deportistas de diferentes especialidades, entre un sprint de $30 \mathrm{~m} \mathrm{y}$ la fuerza máxima producida durante los primeros $30 \mathrm{~ms}$ de la fase concéntrica en un salto vertical.

En otro estudio con jugadores de rugby profesionales, se observó un valor de correlación despreciable y no significativo entre el tiempo en un sprint de $10 \mathrm{~m}$ y el peso levantado en 3RM en squat (Baker \& Nance, 1999). Sin embargo, en el citado trabajo de Wisloff et al. (2004) llevado a cabo con futbolistas de élite, se hallaron unos coeficientes de correlación entre el peso levantado en 1RM en $1 / 2$ squat y el tiempo de aceleración en 10 y 30 m de $-0,94(\rho<0,001)$ y $-0,71(\rho<0,01)$, respectivamente.

También se han realizado estudios en los que se ha investigado la relación entre la fuerza máxima y acciones de lanzamiento. Un coeficiente de correlación alto $(r=0,71$; $\rho<0,01)$ fue hallado en un estudio con 12 jugadoras de voleibol, entre el $1 \mathrm{RM}$ en press de banca y la distancia alcanzada en un lanzamiento desde el pecho de un balón de voleibol desde la posición de sentado (Cronin \& Owen, 2004).

En definitiva, las relaciones entre la fuerza máxima y el rendimiento en acciones explosivas no parecen estar del todo claras, pudiendo estar condicionadas por factores como el nivel de entrenamiento de los sujetos y las medidas analizadas. Por esto, y con la intención de contribuir al conocimiento sobre este tema, el objetivo del presente estudio fue analizar las posibles relaciones entre la fuerza máxima en squat y acciones explosivas de salto, sprint y golpeo de balón. 


\section{Método}

\section{Muestra}

Para la realización del presente estudio se contó con una muestra de 16 estudiantes de Ciencias del Deporte de sexo masculino, con una edad media de 19,6 $\pm 1,7$ años, una estatura media de 1,77 $\pm 0,03 \mathrm{~cm}$, y una masa media de 73,7 $\pm 4,4 \mathrm{~kg}$. Todos ellos estaban habituados a la realización de actividad física de forma regular, pero no al entrenamiento específico de fuerza, por lo que en este contexto se les puede considerar como sujetos con escasa experiencia en el entrenamiento de fuerza.

\section{Instrumentos y materiales}

Los instrumentos y materiales empleados para la realización del presente estudio fueron los siguientes:

o Sistema de medición de fuerzas dinámicas Isocontrol dinámico 3.6 (JLMLi+d, S.L.). Este sistema dispone de un sensor externo que realiza una medición directa del espacio relacionado con el tiempo, obteniéndose también datos de velocidad, aceleración, fuerza y potencia mediante una serie de algoritmos matemáticos. (Precisión del espacio: 0,2 mm; precisión del tiempo: 0,2 $\mu \mathrm{s}$; frecuencia de muestreo: $1000 \mathrm{~Hz}$; rango de medición: 0 a $2 \mathrm{~m}$; máxima aceleración: $120 \mathrm{~m} / \mathrm{s}^{2}$ ). El sistema se conecta a un ordenador, en el cual, mediante el software correspondiente (Isocontrol 3.6wXP) se registran los datos.

o Plataforma de infrarrojos Ergo Jump Bosco System, con 2 barreras de fotocélulas de 1,05 m de longitud, y ordenador Psion.

o Sistema de medición de tiempos Newtest 300 - Series Powertimers, con ordenador de mano Palm Zire con software Newtest Powertimer, 5 fotocélulas y 4 trípodes para colocación de las mismas (sensibilidad: 0,001 s).

o ChronoMaster y sensor de paso multihaz Sportmetrics, con una barrera formada por 10 fotocélulas y una barrera reflectante (sensibilidad: 0,001 s).

o 2 postes metálicos para red de bádminton.

o Panel de madera con las dimensiones de una portería de fútbol sala (3 x 2 m).

o Un balón de fútbol sala Puma Cellerator Sala Oficial. 


\section{Medidas}

Las medidas que se utilizaron para la evaluación de la fuerza máxima en squat fueron:

o 1RM (kg): máximo peso que se puede levantar en una repetición.

o Fuerza media total en 1RM (N): suma de todos los valores de fuerza registrados en una repetición máxima dividido por el tiempo empleado en realizarla (González Badillo \& Ribas, 2002).

o Fuerza dinámica máxima $(\mathrm{N})$ : pico máximo de fuerza obtenido en 1RM (González Badillo \& Ribas, 2002).

o Las medidas que se utilizaron para la evaluación de las acciones explosivas fueron:

o Altura de salto (cm): máxima altura de salto vertical desde parado, con las manos en la cintura y partiendo con un ángulo de flexión de rodillas de $90^{\circ}$ (SJ), con contramovimiento (CMJ) (Bosco, 1994), y con contramovimiento y con la ayuda del movimiento de los brazos (CMJa).

o Tiempo de sprint (s): mejor tiempo de carrera en 5, 10, 15 y $20 \mathrm{~m}$.

o Velocidad de tiro $(\mathrm{m} / \mathrm{s})$ : velocidad media en un golpeo de balón con la máxima potencia.

\section{Procedimiento}

Previamente a la realización de los tests, los sujetos realizaron 4 sesiones en días alternativos (2 sesiones por semana durante 2 semanas) de familiarización y aprendizaje de los tests a ejecutar.

Se solicitó a los participantes que no hicieran ejercicio o actividad física intensa $48 \mathrm{~h}$ antes de las mediciones.

Los tests que se llevaron a cabo se realizaron en dos días diferentes, separados $48 \mathrm{~h}$ entre sí. El primer día se realizó el test de 1RM en squat, y el segundo día los tests de salto, sprint y golpeo de balón. En cada una de las sesiones se realizó un calentamiento adecuado al esfuerzo a llevar a cabo. Los tests que se realizaron consistían en lo siguiente:

Test de 1RM en squat: realización del ejercicio de squat paralelo en una estación Multipower. El peso se incrementaba gradualmente hasta que el sujeto fallaba en una repetición, no pudiendo realizar el ejercicio de manera correcta. El descanso entre repeticiones era aproximadamente de 3 min (Baker, Wilson, \& Carlyon, 1994; Kraemer \& Fry, 1995). De esta manera se obtenía el 1RM, y, mediante el 
sistema Isocontrol Dinámico 3.6, la fuerza media total en 1RM y la fuerza dinámica máxima.

$>$ Tests de salto: se realizaron entre 3 y 5 ensayos consecutivos de cada tipo de salto (SJ, CMJ y CMJa) con el fin de obtener la mayor reproducibilidad posible, utilizando la plataforma de contactos Ergojump Bosco System, y utilizando para el estudio la altura $(\mathrm{cm})$ del mayor salto de cada tipo.

Test de sprint de $20 \mathrm{~m}$ : se llevaron a cabo 3 ensayos no consecutivos de un test propuesto para deportes de equipo (Ellis et al., 2000), utilizando el sistema de registro de Newtest Powertimers, colocándose 5 fotocélulas: en el inicio, a los 5, a los 10, a los 15 y a los $20 \mathrm{~m}$, utilizando para el estudio el mejor tiempo (s) en cada distancia.

> Test de velocidad del balón en el golpeo: se realizaron 4 intentos consecutivos, golpeando con la mayor potencia posible un balón de fútbol sala situado a 6,50 m de un panel de madera con medidas similares a las de una portería de fútbol sala, con la carrera previa, pierna de golpeo y superficie de contacto a libre elección, calculándose el tiempo empleado por el balón en llegar al tablero mediante fotocélulas y sensor de sonido conectadas a la unidad de cronometraje de Sportmetrics. En función de la zona de impacto del balón en el panel los tiempos y distancias eran corregidos para minimizar el error. Para el estudio se utilizó la mayor velocidad $(\mathrm{m} / \mathrm{s})$ de las obtenidas por cada participante.

\section{Análisis estadístico}

Para el análisis estadístico se utilizó el programa SPSS v. 14.0. Se calcularon las medias y desviaciones estándar de las diferentes medidas del estudio. También se calcularon los coeficientes de correlación de Pearson para analizar las posibles relaciones entre las medidas de fuerza máxima en squat y las acciones explosivas, así como su nivel de significación estadística. 
Juarez, D.; Navarro, F.; Aceña, R. M.; González, J.M.; Arija, A.; Muñoz, V. (2008). Relación entre la fuerza máxima en squat y acciones de salto, sprint y golpeo de balón. 10(4), 1-12

\section{Resultados y discusión}

En la tabla 1 se muestran las medias y desviaciones estándar de las medidas empleadas en el presente estudio.

Tabla 1.- Medias y desviaciones estándar de las medidas del estudio

\begin{tabular}{|c|c|c|}
\hline & $\mathrm{N}$ & Media \pm DS \\
\hline IRM (kg) & 16 & $121,04 \pm 19,98$ \\
\hline $\begin{array}{l}\text { Fuerza media total } \\
\qquad(\mathrm{N})\end{array}$ & 16 & $1212,80 \pm 201,59$ \\
\hline $\begin{array}{l}\text { Fuerza dinámica } \\
\text { máxima }(\mathrm{N})\end{array}$ & 16 & $1560,74 \pm 255,77$ \\
\hline SJ $(\mathrm{cm})$ & 16 & $38,21 \pm 4,56$ \\
\hline $\mathrm{CMJ}(\mathrm{cm})$ & 16 & $45,97 \pm 5,47$ \\
\hline $\mathrm{CMJ} a(\mathrm{~cm})$ & 16 & $54,06 \pm 6,82$ \\
\hline $5 \mathrm{~m}(\mathrm{~s})$ & 16 & $1,190 \pm 0,060$ \\
\hline $10 \mathrm{~m}(\mathrm{~s})$ & 16 & $1,947 \pm 0,060$ \\
\hline $15 \mathrm{~m}(\mathrm{~s})$ & 16 & $2,655 \pm 0,065$ \\
\hline $20 \mathrm{~m}(\mathrm{~s})$ & 16 & $3,294 \pm 0,105$ \\
\hline
\end{tabular}

Los coeficientes de correlación de Pearson de las medidas referidas a la fuerza máxima en squat y las alturas de salto, tiempos de sprint y velocidad de tiro, así como su significación estadística, están reflejados en la tabla 2.

Tabla 2.- Correlaciones entre las medidas de fuerza máxima y de fuerza explosiva

\begin{tabular}{|c|c|c|c|c|c|c|c|c|}
\hline & SJ (cm) & $\begin{array}{c}\text { CMJ } \\
(\mathrm{cm})\end{array}$ & $\begin{array}{c}\text { CMJ a } \\
(\mathrm{cm})\end{array}$ & $\begin{array}{c}5 \mathrm{~m} \\
(\mathrm{~s})\end{array}$ & $\begin{array}{c}10 \mathrm{~m} \\
(\mathrm{~s})\end{array}$ & $\begin{array}{c}15 \mathrm{~m} \\
(\mathrm{~s})\end{array}$ & $\begin{array}{c}20 \mathrm{~m} \\
(\mathrm{~s})\end{array}$ & $\begin{array}{c}\text { velocidad } \\
\text { tiro (m/s) }\end{array}$ \\
\hline $1 \mathrm{RM}(\mathrm{kg})$ & $0,62^{*}$ & $0,57 *$ & $0,66 * *$ & $-0,19$ & $-0,24$ & $-0,23$ & $-0,32$ & 0,25 \\
\hline $\begin{array}{c}\text { Fuerza } \\
\text { media } \\
\text { total } \\
(\mathrm{N})\end{array}$ & $0,67 * *$ & $0,59 *$ & $0,67 * *$ & $-0,15$ & $-0,22$ & $-0,20$ & $-0,29$ & 0,28 \\
\hline $\begin{array}{c}\text { Fuerza } \\
\text { dinámica } \\
\text { máxima } \\
(\mathrm{N})\end{array}$ & $0,60 *$ & $0,52 *$ & $0,63 *$ & $-0,06$ & $-0,26$ & $-0,25$ & $-0,30$ & 0,34 \\
\hline
\end{tabular}


Juarez, D.; Navarro, F.; Aceña, R. M.; González, J.M.; Arija, A.; Muñoz, V. (2008). Relación entre la fuerza máxima en squat y acciones de salto, sprint y golpeo de balón. 10(4), 1-12 http://www.cafyd.com/REVISTA/01001.pdf

$* * \rho<0,01 ; \quad * \rho<0,05$

La similitud existente entre el test de squat y los tests de salto en cuanto a la participación muscular y en parte a la biomecánica del gesto, hacen pensar en la posible existencia de un alto grado de relación entre ambas acciones. Sin embargo, los valores de correlación encontrados entre las medidas relativas al 1RM en squat y las medidas de salto son moderados (entre 0,52 y 0,67; algunos significativos al nivel de $\rho<0,01$ y otros al nivel de $\rho<0,05)$ no habiéndose encontrado coeficientes de correlación altos. Un valor de correlación superior $(0,78 ; \rho<0,05)$ a los hallados en nuestro estudio se obtuvo entre la capacidad de salto vertical y el 1RM con futbolistas de élite (Wisloff, Castagna, Helgerud, Jones, \& Hoff, 2004), aunque, a diferencia de nuestro trabajo, en éste se empleo el ejercicio de $1 / 2$ squat, en lugar del squat paralelo como en nuestro caso. En este sentido, es posible que el ángulo de flexión de rodillas del ejercicio de $1 / 2$ squat se asemeje más al empleado en los saltos, lo que puede afectar al coeficiente de correlación de forma positiva. También hay que considerar que el estudio citado se llevó a cabo con sujetos que poseían un cierto nivel de experiencia en el entrenamiento de fuerza, también a diferencia de nuestro estudio, en el que los participantes poseían una escasa experiencia en el trabajo de fuerza. Sin embargo, aunque también empleando el ejercicio de $1 / 2$ squat en lugar de squat, $y$ utilizando para la correlación los valores correspondientes a los cambios producidos tras un periodo de 13 semanas de entrenamiento, en el estudio de Alegre (2004) se encontró un coeficiente de correlación con respecto al SJ $(0,57 ; \rho<0,05)$ parecido a los hallados en la presente investigación.

Como se puede observar en la tabla 2, los coeficientes de correlación del 1RM, fuerza media total y fuerza dinámica máxima, y su significación, con respecto a las alturas de salto, los tiempos de sprint y la velocidad de tiro, son similares, por lo que se calcularon también los coeficientes de correlación entre las tres medidas relacionadas con la fuerza máxima en squat, encontrándose valores muy altos $(>0,9 ; \rho<0,01)$. De esto se deduce que las medidas relacionadas con la fuerza máxima en squat analizadas en este estudio (1RM, fuerza media total y fuerza dinámica máxima) tienen una estrecha relación entre sí.

Los coeficientes de correlación entre las medidas de fuerza máxima en squat y los tiempos de sprint, fueron bajos (entre -0,20 y -0,32) y no significativos, al igual que en otros estudios (Baker \& Nance, 1999; Cronin \& Hansen, 2005). En este caso, habría que tener en cuenta que la aceleración en carrera presenta en su ejecución menos similitudes biomecánicas que el salto vertical con respecto al ejercicio de squat.

En ocasiones, los valores de correlación entre las acciones comentadas anteriormente están influidos por la masa de los sujetos, ya que, por ejemplo, un sujeto puede poseer una gran potencia de salto, pero debido a su peso, no consigue una altura considerable. En el estudio de Baker y Nance (1999) con jugadores profesionales de rugby, el coeficiente de correlación entre el 3RM en squat y el tiempo en $10 \mathrm{~m}$, a pesar de seguir siendo no significativo aumentó, de $-0,06$ a $-0,39$, al tener en cuenta la masa de los jugadores. Por esto, en el presente estudio, también se calcularon los coeficientes de correlación tomando los valores relativos en función de la masa corporal de los sujetos. Sin embargo, en este caso, se encontraron valores similares a los descritos anteriormente, por lo que parece deducirse que, en nuestro estudio, la masa de los sujetos no influye de manera relevante en las relaciones entre las medidas analizadas. 
Por otro lado, si analizamos las correlaciones halladas entre las medidas de fuerza máxima en squat y la velocidad de tiro, se observan valores bajos y no significativos. En este sentido, no se han encontrado referencias de este tipo de correlaciones en la literatura científica. Esta ausencia de relación parece estar relacionada tanto con las diferencias en la biomecánica del gesto y en la participación muscular, como con la gran influencia de la técnica en el golpeo de balón, sobre todo en los participantes en este estudio, muchos de los cuales tenían poca experiencia en esta acción.

En una investigación con 12 futbolistas júnior de élite, no se encontró relación entre la fuerza isocinética y la velocidad de golpeo de ambas piernas (McLean \& Tumilty, 1993). Sin embargo, en otra investigación, la correlación en jóvenes entre fuerzas resultantes máximas de la pantorrilla y pierna y la velocidad de salida del balón era alta (Luhtanen, 1988). Hay autores que han afirmado que la gran velocidad del balón producida por los futbolistas masculinos, en comparación con los femeninos, se debe a su mayor fuerza medida en un dinamómetro isocinético (Tant, Browder, \& Wilkerson, 1991). También hay otros autores que defienden la alta correlación entre la fuerza y la velocidad que se imprime al balón en el golpeo (Cabri, De Proft, Dufour, \& Clarys, 1988; Narici, Sirtori, \& Mognoni, 1988; Poulmedis, Rondoyannis, Mitsou, \& Tsarouchas, 1988). En este sentido, parece, por tanto, que la influencia del trabajo de fuerza en la mejora de los golpeos de balón en fútbol no ha sido aclarada hasta el momento por los científicos del deporte, por lo que se necesita ampliar el conocimiento sobre este tema.

Todo lo comentado anteriormente lleva a plantearse la influencia de la fuerza máxima sobre el rendimiento en acciones como el salto, el sprint o el golpeo de balón. En cualquier caso, hay que considerar que las medidas de fuerza máxima realizadas en este estudio se llevaron a cabo en el ejercicio de squat, cuya mecánica de ejecución y participación muscular no comparte muchos elementos en común, por ejemplo, como ya se ha dicho, con el golpeo de balón. De todas formas, esto no quiere decir que el entrenamiento de la fuerza máxima no sea importante en deportes como el fútbol, donde se pretenden mejorar las acciones explosivas aquí tratadas, ya que una base de fuerza máxima permitirá un mejor desarrollo de la fuerza explosiva requerida en estas acciones, además de ser útil para otras cuestiones, como por ejemplo la prevención de algunas lesiones. En cualquier caso, la muestra de participantes en el presente estudio es reducida, por lo que cualquier interpretación debe ser realizada con cautela, requiriéndose la realización de estudios con un mayor número de sujetos para comprobar si se pueden confirmar los resultados obtenidos en la presente investigación.

\section{Conclusiones}

A la vista de los resultados obtenidos en el presente estudio se puede concluir lo siguiente:

La relación entre la fuerza máxima en squat y la altura de salto vertical en sujetos con escasa experiencia en el entrenamiento de fuerza parece ser de un nivel moderado, mientras que no parece existir relación entre esta fuerza y el tiempo de sprint, ni entre dicha fuerza y la velocidad del balón en el golpeo con el pie. 


\section{Referencias bibliográficas}

Alegre, L. M. (2004). Cambios en la arquitectura y biomecánica del músculo esquelético tras un entrenamiento de fuerza explosiva. Universidad de Castilla La Mancha, Toledo.

Baker, D. (1996). Improving vertical jump performance through general, special, and specific strength training: a brief review. Journal of Strength and Conditioning Research, 10(2), 131-136.

Baker, D. (2001). A series of studies on the training of high-intensity muscle power in rugby league football players. Journal of Strength and Conditioning Research, 15(2), 198-209.

Baker, D., \& Nance, S. (1999). The relation between running speed and measures of strength and power in professional rugby league players. Journal of Strength and Conditioning Research, 13(3), 230-235.

Baker, D., Wilson, G., \& Carlyon, B. (1994). Generality versus specificity: a comparison of dynamic and isometric measures of strength and speed-strength. European Journal of Applied Physiology, 68, 350-355.

Bompa, T. O. (1999). Periodization. Theory and methodology of training (4a ed.). Champaign: Human Kinetics.

Bompa, T. O. (2003). Entrenamiento de la potencia para el fútbol. Extraído el 30 de mayo, 2003, de http://www. sobreentrenamiento.com/PubliCE/Home.asp

Bosco, C. (1994). La valoración de la fuerza con el test de Bosco. Barcelona: Paidotribo.

Bosco, C. (2000). La fuerza muscular. Barcelona: Inde.

Bührle, M., \& Schmidtbleicher, D. (1977). Influence of maximum strength training on the speed of movement. Leistungssport, 7(1), 3-10.

Cabri, J., De Proft, E., Dufour, W., \& Clarys, J. P. (1988). The relation between muscular strength and kick performance. En T. Reilly, A. Lees, K. Davids \& W. J. Murphy (Eds.), Science and Football (Vol. I, pp. 186-193). New York: E \& F. N., Spon.

Cronin, J. B., \& Hansen, K. T. (2005). Strength and power predictors of sports speed. Journal of Strength and Conditioning Research, 19(2), 349-357.

Cronin, J. B., \& Owen, G. J. (2004). Upper-body strength and power assessment in women using a chest pass. Journal of Strength and Conditioning Research, 18(3), 401-404.

Ellis, L., Gastin, P., Lawrence, S., Savage, B., Buckeridge, A., Stapff, A., et al. (2000). Protocols for the physiological assessment of team sport players. En C. J. Gore \& Australian Institute of. Sports (Eds.), Physiological tests for Elite Athletes (pp. 128-144). Champaign: Human Kinetics.

González Badillo, J. J. (2000). Concepto y medida de la fuerza explosiva en el deporte. Posibles aplicaciones al entrenamiento. Revista de Entrenamiento Deportivo, XIV(1), 5-16.

González Badillo, J. J., \& Ribas, J. (2002). Bases de la programación del entrenamiento de fuerza. Barcelona: Inde. 
Juarez, D.; Navarro, F.; Aceña, R. M.; González, J.M.; Arija, A.; Muñoz, V. (2008). Relación entre la fuerza máxima en squat y acciones de salto, sprint y golpeo de balón. 10(4), 1-12 http://www.cafyd.com/REVISTA/01001.pdf

Graham, J. (2002). Periodization research an example application. National Strength and Conditioning Association Journal, 24(6), 62-70.

Häkkinen, K., Komi, P. V., \& Tesch, P. A. (1981). Effect of combined concentric and eccentric strength training and detraining on force-time, muscle fiber and metabolic characteristics of leg extensor muscles. Scandinavian Journal of Sports Sciences, 3(2), 50-58.

Hoff, J., \& Almasbakk, B. (1995). The effects of maximum strength training on throwing velocity and muscle strength in female team-handball players. Journal of Strength and Conditioning Research, 9(4), 255-258.

Kaneko, M., Fuchimoto, T., Toji, H., \& Sney, K. (1983). Training effect of different loads on the force-velocity relationship and mechanical power output human muscle. Scandinavian Journal of Sports Sciences, 5(2), 50-55.

Kraemer, W. J., \& Fry, A. C. (1995). Strength testing: development and evaluation of methodology. En P. J. Maud \& C. Foster (Eds.), Physiological assessment of human fitness (pp. 115-138). Champaign: Human Kinetics.

Luhtanen, P. (1988). Kinematics and kinetics of maximal instep kicking-in junior soccer players. En T. Reilly, A. Lees, K. Davids \& W. J. Murphy (Eds.), Science and Football (Vol. I, pp. 441-448). New York: E \& F. N., Spon.

Manno, R. (1999). El entrenamiento de la fuerza. Barcelona: Inde.

McLean, B. D., \& Tumilty, D. M. (1993). Left-right asymmetry in two types of soccer kick. British J ournal of Sport Medicine, 27(4), 260-262.

Moss, B. M., Refsnes, P. E., Abildgaard, A., Nicolaysen, K., \& Jensen, J. (1997). Effects of maximal effort strength training with different loads on dynamic strength, cross-sectional area, load-power and load-velocity relationships. European J ournal of Applied Physiology, 75, 193-199.

Narici, M. V., Sirtori, M. D., \& Mognoni, P. (1988). Maximal ball velocity and peak torques of hip flexor and knee extensor muscles. En T. Reilly, A. Lees, K. Davids $\&$ W. J. Murphy (Eds.), Science and Football (Vol. I, pp. 429-433). New York: E $\&$ F. N., Spon.

Poulmedis, P., Rondoyannis, G., Mitsou, A., \& Tsarouchas, E. (1988). The influence of isokinetic muscle torque exerted in various speeds on soccer ball velocity. J ournal of Orthopaedic and Sport Physical Therapy, 10(3), 93-96.

Schmidtbleicher, D. (1992). Training for power events. In P. V. Komi (Ed.), Strength and power in sport (pp. 381-395). London: Blackwell Scientific Publications.

Siff, M. C., \& Verkhoshansky, Y. (2000). Superentrenamiento. Barcelona: Paidotribo.

Stone, M. H., O'Bryant, H. S., \& Garhammer, J. (1981). A hypothetical model for strength training. Journal of Sports Medicine, 21, 342-351.

Tant, C. L., Browder, K. D., \& Wilkerson, J. D. (1991). A three-dimensional kinematic comparison of kicking techniques between male and female soccer players. En C. L. Tant, P. E. Patterson \& S. L. York (Eds.), Biomechanis in Sport IX (pp. 101-105). Ames, IA: I owa State University Press.

Voigt, M., \& Klausen, K. (1990). Changes in muscle strength and speed of an unloaded movement after various training programmes. European Journal of Applied Physiology and Occupational Physiology, 60(5), 370-376.

Wathen, D., Baechle, T. R., \& Earle, R. W. (2000). Training variation: Periodization. En T. R. Baechle \& R. W. Earle (Eds.), Essentials of Strength Training and Conditioning. Champaign: Human Kinetics. 
Wenzel, R. R., \& Perfetto, E. M. (1992). The effect of speed versus non-speed training in power development. Journal of Applied Sports Science Research, 6(2), 82-87.

Wilson, G. J., Lyttle, A. D., Ostrowski, K. J., \& Murphy, A. J. (1995). Assessing dynamic performance: a comparison of rate of force development tests. Journal of Strength and Conditioning Research, 9(3), 176-181.

Wisloff, U., Castagna, C., Helgerud, J., Jones, R., \& Hoff, J. (2004). Strong correlation of maximal squat strength with sprint performance and vertical jump height in elite soccer players. British J ournal of Sport Medicine, 38, 285-288.

Young, W. B., \& Bilby, G. E. (1993). The effect of voluntary effort to influence speed of contraction on strength, muscular power and hypertrophy development. Journal of Strength and Conditioning Research, 7(3), 172-178. 